\title{
Identificação de práticas de gestão da segurança e saúde no trabalho em obras de construção civil
}

\author{
Identification of health and safety managerial practices \\ in construction sites
}

\author{
Marcelle Engler Bridi \\ Carlos Torres Formoso \\ Eugenio Pellicer \\ Fabiana Fabro \\ Maria Encarnacion Viguer Castello \\ Marcia Elisa Soares Echeveste
}

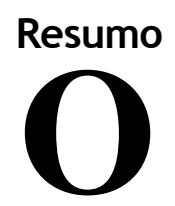

setor da construção civil apresenta índices de acidentes de trabalho mais elevados em relação à maioria dos demais setores industriais. Melhorias nas práticas atuais de gestão da segurança e saúde no trabalho (SST) podem trazer benefícios como a preservação da vida humana e o aumento da competitividade das empresas no mercado com a redução dos custos decorrentes de acidentes, multas e embargos. Este artigo apresenta um levantamento e uma avaliação do grau de implementação das práticas de gestão em SST e a identificação das dificuldades na implementação destas práticas e no cumprimento das normas, na perspectiva dos responsáveis pela gestão da segurança em empresas construtoras. Os dados foram coletados através da realização de uma survey, com questionários aplicados nos canteiros de obra de 40 empresas do segmento de edificações residenciais de Porto Alegre (RS). Estes

Marcelle Engler Bridi Universidade Federal do Rio Grande Porto Alegre - RS - Brasil

Carlos Torres Formoso Universidade Federal do Rio Grande do Sul Porto Alegre - RS - Brasil

Eugenio Pellicer Eugenio Pellicer
Universitat Politècnica de València Valencia - Espanha

Fabiana Fabro Universidade Federal do Rio Grande do Sul
- Brasil Porto Alegre - RS - Brasil

Maria Encarnacion Viguer Catello Universitat Politècnica de València E-mail: mavicas@cst.upv.es

Marcia Elisa Soares Echeveste Universidade Federal do Rio Grande do Sul Porto Alegre - RS - Brasil

Recebido em 06/04/13 Aceito em 11/09/13 dados foram comparados com os resultados de uma survey similar realizada na Espanha com 20 empresas construtoras. Para analisar e classificar as práticas utilizaram-se técnicas estatísticas de análise de componentes principais e múltiplas respostas. Foram criadas cinco categorias de práticas de gestão da SST, sendo analisadas as práticas individuais mais utilizadas na amostra de empresas. Faz-se também uma comparação entre as categorias de práticas mais utilizadas e a percepção de importância por parte dos gerentes.

Palavras-chave: Segurança e saúde no trabalho. Melhores práticas. Gestão. Construção civil.

\section{Abstract}

The construction industry has one of the highest occupational accidents rates in comparison to other industrial sectors. Improvements in current health and safety managerial practices can bring benefits such as preserving human life and increasing companies' competitiveness by the reduction of costs arising from accidents, interdictions, and fines. This article presents a survey on the use of health and safety managerial practices, and on the difficulties for implementing those practices, as well as for being in compliance with safety regulations, from the perspective of the people responsible for safety management in construction companies. Data were collected in the construction sites of 40 construction companies from the residential building sector of Porto Alegre, Brazil. Those data were compared to the results of a similar survey carried out in Spain with 20 construction companies. The principal component analysis, and multiple response analysis techniques were used to analyze and classify the practices. Five categories of safety management practices were proposed, and an analysis of the most used practices in the sample of companies was made. Moreover, a comparison was made between the most adopted categories of practices and the perception of their importance from managers.

Keywords: Occupational safety and health. Best practices. Management. Construction.

BRIDI, M. E.; FORMOSO, C. T.; PELLICER, E.; FABRO, F.; VIGUER CASTELLO, M. E.; ECHEVESTE, M. E. S. Identificação de práticas de gestão da segurança e saúde no trabalho em obras de construção civil. Ambiente Construído, Porto Alegre, v. 13, n. 3, p.43-58, jul./set. 2013.

ISSN 1678-8621 Associação Nacional de Tecnologia do Ambiente Construído. 


\section{Introdução}

A atenção à questão da SST no setor da construção tem aumentado em diversos países nas últimas décadas. No Brasil, em parte, isto é resultado da evolução legislativa combinada com ações de responsabilização trabalhista, penal, previdenciária, civil, administrativa e tributária dos responsáveis pelos danos causados aos trabalhadores (BRASIL, 2010). Na literatura internacional, a visão de que as melhorias na gestão da segurança são investimentos tem crescido na indústria da construção, principalmente devido ao aumento dos custos decorrentes acidentes (ROWLINSON, 2000; GYI; GIBB; HASLAN, 1999; EVERETT; THOMPSON, 1995).

Por outro lado, os dados de inspeção governamental em segurança no trabalho no Brasil apontam que o setor econômico da construção apresenta altos índices de acidentes, embargos e interdições (BRASIL, 2012a). Situações similares têm sido identificadas em outros países. Por exemplo, na Espanha, Díaz, Orden e Zimmermann (2010) apontam que a construção é um dos setores industriais mais importantes, porém com os piores índices de acidentes. Além disso, o gerenciamento da prevenção de riscos não é realizado de maneira eficaz, existindo falhas em medidas de prevenção básicas (DÍAZ; ORDEN; ZIMMERMANN, 2010). Em suma, apesar dos esforços que vem sendo feitos pela indústria e pela academia em distintos países, no sentido de desenvolver inovações tecnológicas e gerenciais relacionadas à SST, Harper e Kohen (1998) apontam que a indústria da construção civil apresenta mais dificuldade em relação a outras indústrias, no que diz respeito à conscientização e à adoção de procedimentos de trabalho seguros. Segundo Asfaw, Pana-Cryan e Rosa (2011), este problema está relacionado às influências da instabilidade econômica neste setor.

A identificação das boas ou melhores práticas de SST tem sido um foco bastante comum em diversos estudos acadêmicos, em diferentes países, principalmente nos Estados Unidos (LISKA; GOODLOE; SEN, 1993; JASELSKI; ANDERSON; RUSSEL, 1996; HINZE, 2002; POTTS; McGLOTHLIN, 2003 ABUDAYYECH et al., 2006), mas também na Grã Bretanha (SAWACHA; NAOUM; FONG, 1999), Austrália (MOHAMED, 2002), China (FANG et al., 2004), Cingapura (TEO; LING; CHONG, 2005) e Chile (RAZURI, 2007). Alguns destes trabalhos realizaram comparações entre as práticas adotadas em empresas de diferentes países (LAI; LIU; LING, 2011). A maior parte dos estudos realizados buscou não somente identificar as práticas de gestão de SST, mas também avaliar o impacto das mesmas na melhoria do desempenho da SST, medido por meio de indicadores de incidência de acidentes.

Contudo, existem algumas limitações nos estudos realizados. Em primeiro lugar, não há uma definição criteriosa sobre o que são práticas e como estas podem ser categorizadas. Alguns dos estudos citados tiveram como foco práticas bem específicas, enquanto outros fazem comparações com base em práticas mais gerais que, de fato, representam categorias de práticas. Além disso, a maioria dos estudos teve seu escopo limitado à investigação em grandes empresas do setor e em países com alto grau de desenvolvimento, tais como os Estados Unidos, por exemplo. Neste sentido, Hinze (2002) afirma que há uma necessidade de se estender esse tipo de estudo às empresas de outros portes e que esse tipo de pesquisa deve ser repetida e atualizada periodicamente.

No Brasil, os estudos de práticas têm focado principalmente em práticas tecnológicas (SAURIN, 1997; SERRA, 2010; KATO; SERRA, 2012; MELO; SANTOS; SERRA, 2012), ou nas práticas adotadas para atender aos requisitos mínimos das normas regulamentadoras de segurança (ROCHA, 1999; MALLMANN, 2008; CAMBRAIA; FORMOSO, 2011), principalmente a NR 18 - Norma Regulamentadora de Condições e Ambiente de Trabalho na Construção Civil (BRASIL, 2012b). O principal objetivo dessas pesquisas tem sido a realização de benchmarking, apontando práticas ou requisitos mínimos que são de mais difícil implementação, muitas vezes apontando as dificuldades enfrentadas pelas empresas na aplicação de algumas práticas. Como os mesmos têm uma ênfase tecnológica, as avaliações são realizadas de forma sistemática através de check-lists, preenchidos através de observações diretas realizadas em canteiros de obras. No caso das práticas de gestão de SST, a coleta é um pouco mais complexa, na medida em que estas necessitam de uma descrição mais complexa para serem avaliadas.

O presente estudo fez parte de um estudo maior sobre a identificação das melhores práticas de gestão de SST no setor da construção (BRIDI, 2012). O objetivo do presente artigo consiste em identificar as práticas mais utilizadas por empresas construtoras do Brasil, e avaliar o grau de implementação destas práticas em uma amostra de empresas do segmento de obras residenciais em Porto Alegre, Brasil. Além disto, foram identificadas as principais dificuldades na

44 Bridi, M. E.; Formoso, C. T.; Pellicer, E.; Fabro, F.; Viguer Castello, M. E.; Echeveste, M. E. S. 
implementação dessas práticas, na perspectiva dos responsáveis pela gestão da SST e dos operários do setor. Também foram identificadas as dificuldades no cumprimento dos requisitos da principal norma regulamentadora de SST, a NR 18 (BRASIL, 2012b), e as principais causas de interdições e embargos nas obras. Com base na visão dos gestores, buscou-se analisar se o não cumprimento estava relacionado a causas gerenciais da empresa, a problemas na legislação ou a outros fatores que poderiam levar a uma discussão acerca de melhorias.

Foi realizada também uma comparação entre as práticas mais utilizadas pela amostra de empresas do Brasil com os resultados de uma amostra de empresas da Espanha. Esta comparação é relevante pelo fato de que ambos os países apresentam algumas similaridades do ponto de vista de tecnologias construtivas utilizadas (por exemplo, estruturas de concreto, paredes de alvenaria, revestimentos de argamassa), mas possuem marcos institucionais e legais bastante distintos. De fato, a normalização relativa à segurança e saúde no trabalho da Espanha passou por uma profunda mudança nas últimas décadas, contribuindo para que a indústria da construção daquele país tivesse uma expressiva melhora no seu desempenho em relação à incidência de acidentes do trabalho (MARTINEZ; RUBIO; GIBB, 2010).

\section{Boas práticas de gestão da SST}

Não existe na literatura sobre gestão de SST uma conceituação clara sobre o que é um prática ou melhor prática de SST. De fato, existem divergências nas definições adotadas por diferentes autores: enquanto alguns estudos utilizam o termo boas ou melhores práticas (RAZURI, 2007; LAI; LIU; LING, 2011), ou também técnicas (LISKA; GOODLOE; SEN, 1993; REESE, 1999) e elementos (JASELSKI; ANDERSON; RUSSEL, 1996; POTTS; MCGLOTHLIN , 2003). No que diz respeito ao enfoque dos estudos realizados, aparecem termos como fatores (SAWACHA; NAOUM; FONG, 1999; FUNG et al., 2005; TEO; LING; CHONG, 2005) e constructos (MOHAMED, 2002), por exemplo, os quais também fazem referência a práticas singulares (FAMÁ, 2010; HUANG; HINZE, 2006a, 2006b) ou definem características de um conjunto de práticas (categorias de práticas) (HINZE, 2002).

Um ponto comum nos trabalhos anteriores é que conceito do que é uma boa ou melhor prática está relacionado com a contribuição da implementação da prática na melhoria do desempenho da SST. Entretanto, uma deficiência encontrada em grande parte da literatura sobre este tema é que não é levado em conta que a melhoria no desempenho não é resultado de uma única prática, mas da implementação de um conjunto de práticas que levam em conta uma série de fatores contextuais, os quais são únicos para cada situação (BRIDI, 2012). Ou seja, as práticas não tem um impacto intrinsecamente positivo, mas o resultado depende do contexto, tais como os recursos disponíveis para implantação da mesma e a capacitação daqueles que a aplicam. Assim, optou-se por utilizar neste estudo apenas o termo "práticas de gestão da SST".

Este estudo adota o seguinte conceito de prática de gestão da SST: é um processo gerencial, que pode empregar uma ou mais técnicas e ferramentas, seja ela obrigatória pela legislação ou voluntária, cujo objetivo é contribuir para o controle de riscos relacionados à SST. Essa definição foi proposta a partir de uma discussão realizada em conjunto com pesquisadores de uma rede de pesquisa denominada GESST-IC ${ }^{1}$.

Embora não haja um consenso quanto ao nome, tipo e número de categorias de práticas de gestão de SST, algumas categorias de práticas são mais investigadas pela comunidade acadêmica, como é o caso das categorias "Comprometimento da gerência com a SST", "Treinamentos", "Planejamento da SST", "Programas de Incentivo", "Envolvimento dos trabalhadores" e "Gestão dos Subempreiteiros". Algumas destas práticas são inclusive explicitamente citadas por normas de gestão da segurança tais como a OHSAS 18001 (BRITISH..., 2007).

Ainda que a maioria das práticas possa ser classificada em mais de uma categoria na gestão da segurança, para melhor compreensão é necessário definir critérios que permitam a categorização dessas práticas. Para tal, foram selecionadas categorias mais frequentemente utilizadas na literatura, que se adequavam às práticas encontradas nas empresas investigadas. Assim, para realização deste estudo, foram selecionadas 27 práticas, organizadas em 5 categorias, as quais são descritas na sequência. Essas práticas foram identificadas tanto na revisão da literatura, e também apontadas como relevantes para o contexto brasileiro nas entrevistas realizadas com

\footnotetext{
${ }^{1}$ A Rede Temática GESST-IC (Gestão da Segurança e Saúde no Trabalho na Indústria da Construção: novas abordagens e de benchmarking) foi formada por nove universidades de sete países (Brasil, Chile, Colômbia, México, Uruguai, Portugal e Espanha), através do financiamento do CYTED (Programa IberoAmericano de Ciência e Tecnologia para o Desenvolvimento), através do projeto $n^{\circ} 309$ RT0375. O principal objetivo dessa rede é estudar as melhores práticas de gestão da SST implementadas nas empresas líderes em países ibero-americanos (FORMOSO; PELLICER; YEPES, 2011).
} 
os gestores das construtoras participantes do presente trabalho (ver método de pesquisa).

\section{Planejamento da SST}

Corresponde às ações de planejamento, realizado em todas as etapas do empreendimento, para que as tarefas sejam realizadas com segurança, integrando a gestão à rotina de trabalho, assim como realizando planos específicos para cada obra e os atualizando. O planejamento deve ser feito para eliminar perigos ou estabelecer medidas preventivas para os mesmos, assim como permitir que os recursos (por exemplo, materiais e equipamentos de SST) estejam disponíveis no canteiro quando necessários para a execução das tarefas (REESE, 1999).

Liska, Goodloe e Sen (1993) dividem o planejamento em duas principais ações: préconstrução e pré-tarefa. Segundo os autores, durante a pré-construção, antes do início da etapa de construção, deve-se reunir todos os envolvidos e identificar os recursos materiais necessários para o cumprimento do programa de segurança. Já o planejamento pré-tarefa, segundo os referidos autores, consiste em identificar os perigos antes de iniciar cada tarefa.

\section{Controle da SST}

Para Razuri (2007), o desempenho da SST está relacionado ao grau de precisão e detalhe com que se planeja, organiza e controla a execução das atividades. $O$ controle pode ser realizado através do monitoramento e da realização de medições de desempenho. Segundo Lai, Liu e Ling (2011), medições efetivas da gestão da SST podem influenciar o comportamento dos trabalhadores no canteiro. De fato, os resultados das medições de desempenho podem influenciar no clima de segurança da obra, principalmente quando estão relacionados aos programas de incentivos, fazendo com que os trabalhadores colaborem e cobrem uns aos outros pela melhoria dos índices de desempenho (LAI; LIU; LING, 2011). Além disso, realizar medições faz com que os gestores possam monitorar o desempenho do empreendimento, servindo de base para a proposição de melhorias nas técnicas e procedimentos de SST (ABUDAYYECH et al., 2006).

\section{Participação dos trabalhadores}

Os empregados devem ser incentivados a se envolver nas decisões que os afetam diretamente, visando a aumentar o comprometimento e a percepção de que há certo controle em relação àquilo que os impacta (REESE, 1999), ou seja, os mesmos tendem a se sentir motivados a executar algo que ajudaram a elaborar (HARPER; KOEHN, 1998).

Ao adaptar o trabalho ao indivíduo, por exemplo, através da participação do trabalhador, com a escolha conjunta dos métodos e equipamentos a serem utilizados, a empresa oferece meios para melhorar as condições de trabalho dos funcionários (EUROPEAN... 2011).

\section{Realização de treinamentos}

Um treinamento eficaz dos operários e dos supervisores pode contribuir para a conscientização sobre as causas dos acidentes e sobre os benefícios de um bom sistema de gestão da segurança na obra (SAWACHA; NAOUM; FONG, 1999). Harper e Koehn (1998) apontam que a realização de treinamentos transmite a importância conferida à SST pela empresa e a necessidade de comprometimento por parte dos trabalhadores. Treinamentos também desenvolvem habilidades, requisitos essenciais para melhorar o desempenho em relação à gestão da segurança (REESE, 1999).

\section{Contratação de pessoal especializado em SST}

Para Hinze (2002), a presença de profissionais especializados em SST em tempo integral na obra é importante para verificar se as necessidades de SST estão sendo atendidas, devendo haver uma proporção entre a quantidade de especialistas contratados e o número de trabalhadores na obra. Sawacha, Naoum e Fong (1999) destacam o papel destes profissionais na realização de ações preventivas.

Alguns estudos vão além, apontando a necessidade de criação de um setor específico na empresa para coordenar a gestão da SST (SAWACHA; NAOUM; FONG, 1999; JASELSKI; ANDERSON; RUSSEL, 1996; ABUDAYYECH et al., 2006). Para Abudayyech et al. (2006), o coordenador da SST deve ter habilidades e conhecimento para construir uma cultura de SST na empresa e dimensionar o tempo empregado na gestão da SST, podendo este influenciar o desempenho da empresa na prevenção de riscos de acidentes (JASELSKI; ANDERSON; RUSSEL, 1996).

\section{Método de pesquisa}

A survey foi realizada entre os meses de setembro de 2010 e junho de 2011 no Brasil e de março a

46 Bridi, M. E.; Formoso, C. T.; Pellicer, E.; Fabro, F.; Viguer Castello, M. E.; Echeveste, M. E. S. 
maio de 2011 na Espanha, sendo estruturada em duas principais etapas: qualitativa e quantitativa.

O passo inicial da survey, conforme sugerido por Malhotra (2006), é a realização de uma coleta de informações qualitativas, junto a um pequeno número de entrevistados, que permitam coletar informações preliminares sobre $\mathrm{o}$ contexto estudado. Esta etapa foi realizada através de entrevistas semi-estruturadas junto a profissionais de quatro empresas de diferentes portes da cidade de Porto Alegre, Brasil (Quadro 1), as quais eram reconhecidas no meio técnico por terem um bom sistema de gestão da segurança. Optou-se por delimitar o estudo às obras residenciais de tipologia vertical, dada a importância deste tipo de obra no setor da construção civil do pais.

O estudo qualitativo teve como objetivo entender o funcionamento da gestão da SST nas empresas e identificar, entre as práticas indicadas na bibliografia, aquelas que eram mais comuns ao segmento de obras imobiliárias do Brasil. Buscouse também, nestas entrevistas, obter a percepção dos entrevistados quanto às dificuldades na implementação das práticas sugeridas pela bibliografia no país.

Com base na pesquisa qualitativa foi elaborado o questionário utilizado como instrumento de coleta de dados. Assim, foram selecionadas 27 práticas, organizadas em cinco categorias: Planejamento da SST, Controle da SST, Treinamentos, Contratação de Pessoal Especializado e Participação dos Trabalhadores. Algumas exemplos de práticas constam nas figuras e tabelas apresentadas no presente trabalho. Uma relação completa das mesmas está apresentada em Bridi (2012).

A ferramenta de coleta desenvolvida para esta etapa foi estruturada em seis partes principais:

(a) identificação da empresa e do respondente;

(b) identificação das práticas e do grau de implementação;

(c) percepção da importância das categorias de práticas selecionadas; (d) identificação de dificuldades na implantação;

(e) identificação de dificuldades no atendimento aos requisitos da NR 18 (ABNT, 2012b); e

(f) identificação das principais causas de multas, interdições e embargos.

Para avaliar o grau de utilização das práticas, inicialmente o respondente era questionado quanto à existência das 27 práticas selecionadas na empresa, organizadas nas categorias e contendo três opções de respostas (sim/não/não sei). $\mathrm{Na}$ sequência, foram criadas cinco escalas de avaliação do grau de conhecimento ou de implementação das práticas pelo respondente. A Figura 1 mostra parte do questionário com as práticas listadas na categoria "Planejamento da Segurança".

O questionário continha também uma pergunta aberta, na qual o respondente poderia relatar alguma prática de gestão de SST que a empresa adotava, mas não estava incluída na listagem.

A segunda parte teve como objetivo verificar se as categorias de práticas implementadas eram condizentes com as consideradas como de maior importância pelos envolvidos na gestão da SST. Assim, solicitou-se aos respondentes que ordenassem as cinco categorias de práticas, de acordo com o grau de importância que eles atribuíam para cada uma delas (Figura ).

Em relação às dificuldades de implantar as práticas de gestão da SST, foram selecionadas seis alternativas, identificadas nas entrevistas com os especialistas, com uma escala Likert de cinco opções, conforme a Figura .

A mesma estratégia foi utilizada para a identificação das dificuldades no cumprimento das exigências da NR 18 (ABNT, 2012b). Por fim, para proposta de soluções, é necessário conhecer as principais causas de não cumprimento das normas. Assim, os entrevistados selecionavam, entre os 18 itens da NR 18 (ABNT, 2012b), aqueles relacionados com multas, interdições e embargos ocorridos nos empreendimentos.

\section{Quadro 1 - Entrevistas realizadas na fase inicial da pesquisa survey}

\begin{tabular}{|c|c|c|c|c|}
\hline Empresa & Descrição & Função do(s) Entrevistado(s) & Data & Duração \\
\hline A & $\begin{array}{c}\text { Construtora de pequeno porte } \\
\text { e atuação local }\end{array}$ & Engenheiro de Planejamento & $20 / 10 / 2010$ & 43 min \\
\hline B & $\begin{array}{c}\text { Construtora de pequeno porte } \\
\text { e atuação local }\end{array}$ & Gerente de Produção & $22 / 10 / 2010$ & $30 \mathrm{~min}$ \\
\hline C & $\begin{array}{c}\text { Construtora de médio porte e } \\
\text { atuação regional }\end{array}$ & Engenheiro de SST & $25 / 10 / 2010$ & $90 \mathrm{~min}$ \\
\hline D & $\begin{array}{c}\text { Construtora de grande porte e } \\
\text { atuação no MERCOSUL }\end{array}$ & $\begin{array}{c}\text { Engenheiro de SST e Técnico } \\
\text { em SST }\end{array}$ & $26 / 10 / 2010$ & $70 \mathrm{~min}$ \\
\hline
\end{tabular}


Figura 1 - Extrato do questionário - levantamento da implantação

\begin{tabular}{|c|c|c|c|c|c|c|c|c|c|}
\hline \multicolumn{10}{|c|}{ PRÁTICAS UTILIZADAS NA GESTÃO DA SST } \\
\hline \multicolumn{10}{|c|}{14 Em relação à utilização das seguintes práticas de gestão da SST nesta obra: } \\
\hline & 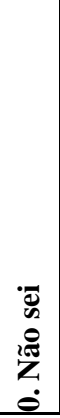 & 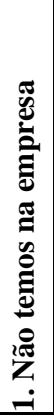 & 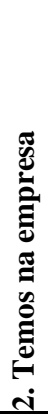 & 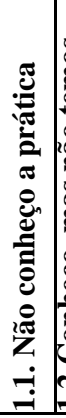 & 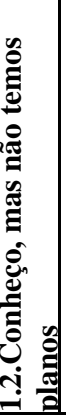 & 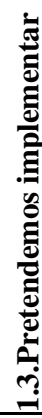 & 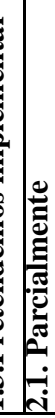 & 矛 & 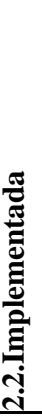 \\
\hline \multicolumn{10}{|l|}{ Planejamento da segurança } \\
\hline \multicolumn{10}{|l|}{$\begin{array}{l}\text { 14.1 Realização de análise de restriçõos de SST no } \\
\text { planejamento (ex. no médio prazo) }\end{array}$} \\
\hline \multicolumn{10}{|l|}{ 14.2 Realização de Análise Preliminar de Riscos } \\
\hline $\begin{array}{l}\text { 14.3 Atualização do PCMAT durante a obra com base nas } \\
\text { mudanças de layout e fases de execução da obra }\end{array}$ & & & & & & & & & \\
\hline
\end{tabular}

Figura 2 - Extrato do questionário - importância das categorias

\begin{tabular}{|l|}
\hline 16 Na tua opinião, em relação às categorias de práticas listadas, ordene \\
as que mais impactam no desempenho da SST? (redução de acidentes, \\
aumento da segurança...)
\end{tabular}

Figura 3 - Extrato do questionário - dificuldades na implementação

18 Leia cada afirmativa e marque com um $X$ a opção relativa à sua concordância em relação às dificuldades de implementação das práticas de Saúde e Segurança no canteiro de obras:

\begin{tabular}{|c|c|c|c|c|c|}
\hline & 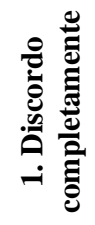 & 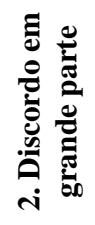 & 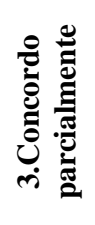 & 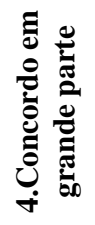 & 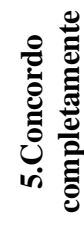 \\
\hline $\begin{array}{l}\text { O aumento dos gastos da construtora é uma dificuldade para } \\
\text { implementação das práticas de SST }\end{array}$ & & & & & \\
\hline $\begin{array}{l}\text { A baixa escolaridade da mão-de-obra é uma dificuldade para } \\
\text { implementação das práticas de SST }\end{array}$ & & & & & \\
\hline $\begin{array}{l}\text { A falta de disponibilidade de profissionais da área de SST no } \\
\text { mercado é uma dificuldade para implementação das práticas de SST }\end{array}$ & & & & & \\
\hline $\begin{array}{l}\text { A grande rotatividade da mão de obra é uma dificuldade para } \\
\text { implementação das práticas de SST }\end{array}$ & & & & & \\
\hline $\begin{array}{l}\text { A queda de produtividade da obra a curto prazo é uma dificuldade } \\
\text { para implementação das práticas de SST }\end{array}$ & & & & & \\
\hline $\begin{array}{l}\text { A resistência das subempreiteiras em se adequar as políticas da } \\
\text { empresa é uma dificuldade para implementação das práticas de SST }\end{array}$ & & & & & \\
\hline
\end{tabular}

48 Bridi, M. E.; Formoso, C. T.; Pellicer, E.; Fabro, F.; Viguer Castello, M. E.; Echeveste, M. E. S. 
Para realizar o plano amostral, optou-se por delimitar a pesquisa às empresas atuantes na Região Metropolitana de Porto Alegre, Brasil, vinculadas ao Sindicato das Indústrias da Construção Civil do Estado do Rio Grande do Sul (SINDUSCON-RS) e com empreendimentos de construção para fins residenciais multi-familiares. Esse critério é decorrente da acessibilidade aos dados. Salienta-se que a opção por um tipo de uso, principalmente no caso dos empreendimentos residenciais, não sendo possível inferir sobre outros segmentos de mercado, como por exemplo, em alguns segmentos, como é o caso de obras industriais. Essas empresas tendem a possuir sistemas de gestão da SST mais completos, principalmente em função do elevado grau de exigência por parte de alguns contratantes de obras (SAURIN, 2002).

O tamanho da amostra para análise estatística foi calculado pelo software WINPEPI v11.1 ${ }^{\circledR}$ (2006). Para um erro relativo de estimação (๕) de $10 \%$ e um intervalo de confiança de $90 \%(1-\alpha=0,90)$, sabendo que há na população $(\mathrm{N})$ considerada de 215 construtoras associadas ao SINDUSCON-RS em Porto Alegre, o tamanho mínimo (n) calculado foi de 38 construtoras. A aplicação do questionário foi realizada por uma equipe de dez graduandos em Estatística e duas mestrandas em Engenharia Civil $^{2}$. A coleta de dados foi realizada diretamente nos canteiros de obras, sendo entrevistados os seguintes profissionais: engenheiros residentes, técnicos de segurança, mestres-de-obras e encarregados de equipes de subempreiteiros. O objetivo de entrevistar profissionais distintos era verificar a existência de diferenças na percepção dos entrevistados e, em paralelo, obter uma maior veracidade na coleta, através da análise comparativa das respostas. Questões gerenciais, por exemplo, eram mais bem respondidas pelos engenheiros de obra, pois estes tinham mais contato com a empresa, enquanto questões operacionais muitas vezes não eram de conhecimento deste profissional, pois, na maioria das empresas, havia certa independência ou terceirização dos técnicos de SST. Assim, as respostas foram analisadas de forma cruzada e os dados foram inseridos em um banco de dados e analisados através de técnicas estatísticas de análise de componentes principais e múltiplas respostas. Também, realizou-se o teste Alfa de Cronbach $\left(\alpha_{\mathrm{s}}\right)$ para avaliar a consistência interna do instrumento de coleta de dados. Este indicador $\left(\alpha_{\mathrm{s}}\right)$ apontou um valor de 0,89 nas questões

\footnotetext{
${ }^{2}$ A survey foi desenvolvida na disciplina Pesquisa e Análise de Mercado, ministrada pelas professoras Márcia Echeveste e Liane Werner do Instituto de Matemática da UFRGS.
}

relativas à implementação das práticas, o que indicou uma consistência interna adequada.

No caso da Espanha, o estudo qualitativo foi realizado com três especialistas na prevenção de riscos no setor da construção. Com base nessa etapa, foram realizadas pequenas alterações no questionário desenvolvido no Brasil. Posteriormente, foram selecionadas 20 empresas entre as líderes no mercado, sendo dez com atuação a nível nacional e dez regionais. Os questionários foram aplicados por pesquisadores da Universidade Politécnica de Valência que entrevistaram os responsáveis pela prevenção de acidentes e os mestres de obras nas empresas colaboradoras com a pesquisa.

\section{Resultados}

\section{Práticas de gestão em SST utilizadas nas empresas}

A Tabela 1 apresenta o ranking das práticas com maior percentual de utilização do total de 27 práticas relacionadas. No caso brasileiro, de 39 empresas respondentes, $80 \%$ utilizavam a prática "Contratação de empresa especializada para elaboração dos projetos das instalações de segurança". Por outro lado, no caso espanhol, três práticas são utilizadas por todas as construtoras investigadas. Ainda, entre as seis práticas com maior percentual em cada país, apenas a prática "A empresa utiliza checklist para conferência e controle de documentação dos subempreiteiros" foi comum entre os países, sendo implementada em $67,5 \%$ das empresas brasileiras e $95 \%$ das espanholas.

As práticas com menor percentual de utilização nos países participantes dessa pesquisa são apresentadas na Tabela 2. Nas empresas construtoras brasileiras, duas práticas obtiveram o menor percentual de utilização (20\%): "Os membros da CIPA participam ativamente da gestão da segurança" e "A empresa possui Programa de Participação nos Resultados, que estimula metas de desempenho da SST". No caso espanhol, quatro das práticas listadas não são implementadas por nenhuma das 20 empresas investigadas. Ainda, em relação à prática "Os responsáveis pela SST podem notificar e paralisar a obra no caso de problemas com a SST", nenhuma das empresas espanholas respondeu que adota a mesma, embora, pela legislação espanhola, tanto o Coordenador da Segurança pode interromper as atividades, como os trabalhadores podem se recusar a realizar as tarefas no caso de considerarem que há situações de falta de segurança. Entre as práticas menos implementadas 
nos dois países, destacam-se a "Realização de programas 5S" e a "Realização de reuniões diárias para tratar da segurança".

Verificou-se que a maior parte das práticas utilizadas pelas empresas brasileiras está diretamente relacionada ao cumprimento da legislação, tais como contratação de projetos específicos, realização e atualização do Programa de Condições e Meio Ambiente do Trabalho (PCMAT), e o controle da documentação dos trabalhadores, por exemplo.

Tabela 1 - Percentual de utilização das práticas mais implementadas no Brasil e na Espanha

\begin{tabular}{|c|c|}
\hline Práticas - Brasil & $\begin{array}{c}\text { TOTAL } \\
\text { n }(\%)\end{array}$ \\
\hline $\begin{array}{l}\text { Contratação de empresa especializada para captação dos projetos das instalações de } \\
\text { segurança. }\end{array}$ & $39(80,0)$ \\
\hline $\begin{array}{l}\text { Empresa especializada em engenharia de segurança e coordenação de equipe de técnicos em } \\
\text { obra. }\end{array}$ & $39(77,5)$ \\
\hline Elaboração periódica de relatório de avaliação da SST, contendo indicadores pró-ativos. & $40(70,0)$ \\
\hline $\begin{array}{l}\text { Atualização do PCMAT durante a obra com base nas mudanças de layout e fases de execução } \\
\text { da obra. }\end{array}$ & $40(67,5)$ \\
\hline A empresa utiliza checklist para conferência e controle de documentação dos subempreiteiros. & $40(67,5)$ \\
\hline Elaboram-se e disponibilizam-se no canteiro de obras instruções de trabalho por função. & $40(62,5)$ \\
\hline Práticas - Espanha & $\begin{array}{c}\text { TOTAL } \\
\text { n }(\%) \\
\end{array}$ \\
\hline Avaliação de riscos & $20(100)$ \\
\hline $\begin{array}{l}\text { Atualização do plano de Segurança e Saúde durante a construção com base na evolução da } \\
\text { execução da obra. }\end{array}$ & $20(100)$ \\
\hline $\begin{array}{l}\text { Contratação de empresa especializada para assessoria em engenharia de segurança e } \\
\text { coordenação da equipe de técnicos de segurança em obra }\end{array}$ & $20(100)$ \\
\hline Possuem procedimentos de SST padronizados que definem como agir em caso de emergência. & $20(95,0)$ \\
\hline A empresa utiliza checklist para conferência e controle de documentação dos subempreiteiros. & $20(95,0)$ \\
\hline Aplicação de listas de verificação das medidas de segurança adotadas na obra. & $20(95,0)$ \\
\hline
\end{tabular}

Nota: $\mathrm{n}$ é o numero de empresas respondentes.

Tabela 2 - Percentual de utilização das práticas menos implementadas no Brasil e na Espanha

\begin{tabular}{l|c}
\hline \multicolumn{1}{c|}{ Práticas - Brasil } & TOTAL \\
\cline { 2 - 2 } & n (\%) \\
\hline Realizam algum evento com enfoque na SST, além da SIPAT. & $40(32,5)$ \\
\hline A Empresa incentiva e treina os funcionários para adotarem a prática dos 5s. & $40(25)$ \\
\hline Reunião diária específica para planejamento da segurança. & $40(22,5)$ \\
\hline Os membros da CIPA participam ativamente da gestão da segurança. & $40(20,0)$ \\
\hline $\begin{array}{l}\text { A empresa possui Programa de Participação nos Resultados, que estimula metas de } \\
\text { desempenho da SST. }\end{array}$ & $40(20,0)$ \\
\hline $\begin{array}{l}\text { Possuem políticas para lidar com funcionários que apresentam problemas com o consumo } \\
\text { de álcool e/ou drogas. }\end{array}$ & $40(20)$ \\
\hline $\begin{array}{l}\text { Possuem políticas para lidar com funcionários que apresentam problemas com o consumo } \\
\text { de álcool e/ou drogas. }\end{array}$ & TOTAL \\
\hline A Empresa incentiva e treina os funcionários para adotarem a prática dos 5S. & n (\%) \\
\hline Possuem técnico de segurança em tempo integral por obra. & $20(10,0)$ \\
\hline Realização de análise de restrições de SST no planejamento (ex. no médio prazo). & $20(0)$ \\
\hline $\begin{array}{l}\text { Os responsáveis pela SST podem notificar e paralisar a obra no caso de problemas com a } \\
\text { SST. }\end{array}$ & $20(0)$ \\
\hline Reunião diária específica para planejamento da segurança. & $20(0)$ \\
\hline
\end{tabular}

50 Bridi, M. E.; Formoso, C. T.; Pellicer, E.; Fabro, F.; Viguer Castello, M. E.; Echeveste, M. E. S. 
Em relação à pergunta aberta, no Brasil foram relatadas as seguintes práticas: "Avaliação periódica da SST, realizada por profissionais externos ao canteiro" (citada por cinco entrevistados); "Visitas e palestras de profissionais da área da saúde" (três citações); "Treinamento para emergências" (duas citações) e "Procedimentos de arrumação e limpeza" (uma citação).

\section{Comparação das práticas mais implementadas com o grau de importância}

Com o objetivo de verificar se as categorias de práticas implementadas são condizentes com as consideradas como de maior importância pelos envolvidos na questão da gestão da SST, solicitouse aos respondentes que ordenassem as cinco categorias de práticas, de acordo com o grau de importância que eles atribuíam para cada categoria. Usando o método descritivo de múltiplas respostas, classificou-se dentro de cada critério: o nível de implementação na empresa (Tabela 3) e a importância das categorias na opinião dos entrevistados (Tabela 4) no Brasil e na Espanha, na Tabela 5 e na Tabela 6 , respectivamente.

Tabela 3 - Ranking das categorias de práticas mais utilizadas no Brasil $^{3}$

\begin{tabular}{l|c|c|c|c|c}
\hline \multicolumn{1}{c|}{ Categorias } & $\mathbf{n}$ & \% Total & $\mathbf{N}^{\mathbf{0}}$ de práticas & \% Média & Ranking \\
\hline $\begin{array}{l}\text { Contratação de pessoal especializado em } \\
\text { segurança }\end{array}$ & 71 & 229,0 & 3 & 76,33 & $\mathbf{1}$ \\
\hline Realização de treinamentos & 29 & 161,1 & 3 & 53,70 & $\mathbf{2}$ \\
\hline Planejamento da segurança & 85 & 314,8 & 6 & 52,47 & $\mathbf{3}$ \\
\hline Controle da segurança & 157 & 448,6 & 10 & 44,86 & $\mathbf{4}$ \\
\hline Participação dos funcionários & 28 & 200,0 & 5 & 40,00 & $\mathbf{5}$ \\
\hline
\end{tabular}

Tabela 4 - Ranking das categorias de práticas mais importantes no Brasil

\begin{tabular}{l|c|c|c}
\hline \multicolumn{1}{c|}{ Categorias } & n & \% Total & Ranking \\
\hline Planejamento da segurança & 9 & $45,0 \%$ & $\mathbf{1}$ \\
\hline Realização de treinamentos & 5 & $25,0 \%$ & $\mathbf{2}$ \\
\hline Participação dos funcionários & 2 & $10,0 \%$ & $\mathbf{3}$ \\
\hline Controle da segurança & 2 & $10,0 \%$ & $\mathbf{3}$ \\
\hline Contratação de pessoal especializado em segurança & 2 & $10,0 \%$ & $\mathbf{3}$ \\
\hline
\end{tabular}

Tabela 5 - Ranking das categorias de práticas mais utilizadas na Espanha

\begin{tabular}{l|c|c|c|c|c}
\hline \multicolumn{1}{c|}{ Categorias } & n & \% Total & $\mathbf{N}^{\mathbf{0}}$ de práticas & \% Média & Ranking \\
\hline Planejamento da segurança & 93 & 465 & 6 & 77,5 & $\mathbf{1}$ \\
\hline Controle da segurança & 167 & 835 & 10 & 69,6 & $\mathbf{2}$ \\
\hline Participação dos funcionários & 32 & 160 & 3 & 53,3 & $\mathbf{3}$ \\
\hline Realização de treinamentos & 26 & 130 & 3 & 43,3 & $\mathbf{4}$ \\
\hline $\begin{array}{l}\text { Contratação de pessoal especializado em } \\
\text { segurança }\end{array}$ & 28 & 140 & 5 & & \\
\hline
\end{tabular}

Tabela 6 - Ranking das categorias de práticas mais importantes na Espanha

\begin{tabular}{l|c|c|c}
\hline \multicolumn{1}{c|}{ Categorias } & n & \% Total & Ranking \\
\hline Planejamento da segurança & 24 & 61,5 & $\mathbf{1}$ \\
\hline Controle da segurança & 7 & 17,9 & $\mathbf{2}$ \\
\hline Participação dos funcionários & 6 & 15,4 & $\mathbf{3}$ \\
\hline Realização de treinamentos & 1 & 2,6 & $\mathbf{4 , 5}$ \\
\hline Contratação de pessoal especializado em segurança & 1 & 2,6 & $\mathbf{4 , 5}$ \\
\hline
\end{tabular}

\footnotetext{
${ }^{3}$ A variável " $n$ " representa a soma das práticas utilizadas pelas empresas dentro de cada categoria, cujo percentual de utilização é apresentado em \%Total. A \%Média representa a divisão de \%Total pelo $\mathrm{N}^{\circ}$ de Práticas de cada categoria, de forma a equilibrar os resultados obtidos e permitir a comparação.
} 
Analisando de forma comparativa, no caso do Brasil, percebe-se que a categoria "Contratação de pessoal especializado" é a mais utilizada pelas empresas, porém a mesma categoria foi ordenada como a de menor importância entre as demais. Esse dado pode ser um indicativo da facilidade que as empresas encontram em terceirizar a gestão da SST. Embora a terceirização de atividades associadas à gestão da SST não seja necessariamente um problema, isso pode ser negativo na medida em que as construtoras subcontratem atividades gerenciais críticas e que devem estar integradas na rotina gerencial, tais como o planejamento e controle da SST. Em relação ao técnico de segurança do trabalho, 35\% das empresas possuem técnicos de SST em tempo integral nas obras, porém, destes, apenas $27 \%$ são funcionários próprios (não terceirizados) das empresas.

Esse resultado não se repete na investigação espanhola, cujos resultados indicam uma maior coerência entre a percepção de importância e a implementação das práticas. Além disso, a categoria de "Planejamento da SST" foi considerada como a de maior importância para a gestão da SST pelos dois países participantes e, da mesma forma, ambos indicaram a "Contratação de pessoal especializado na SST" como de menor importância.

\section{Dificuldades em implementar práticas de SST}

A Figura 4 e a Figura 5 apresentam os percentuais de concordância com as questões investigadas.

No caso brasileiro, a maior dificuldade encontrada, na percepção dos entrevistados, é a resistência dos subempreiteiros, enquanto na Espanha é a grande rotatividade da mão de obra. Em ambos os países a falta de profissionais especialistas na SST obteve o menor percentual de concordância, sendo que 7,5\% dos entrevistados no Brasil concordam em parte ou totalmente e na Espanha nenhum dos entrevistados respondeu concordar com a hipótese verificada.

Figura 4 - Dificuldades na implementação das práticas de gestão da SST - Brasil

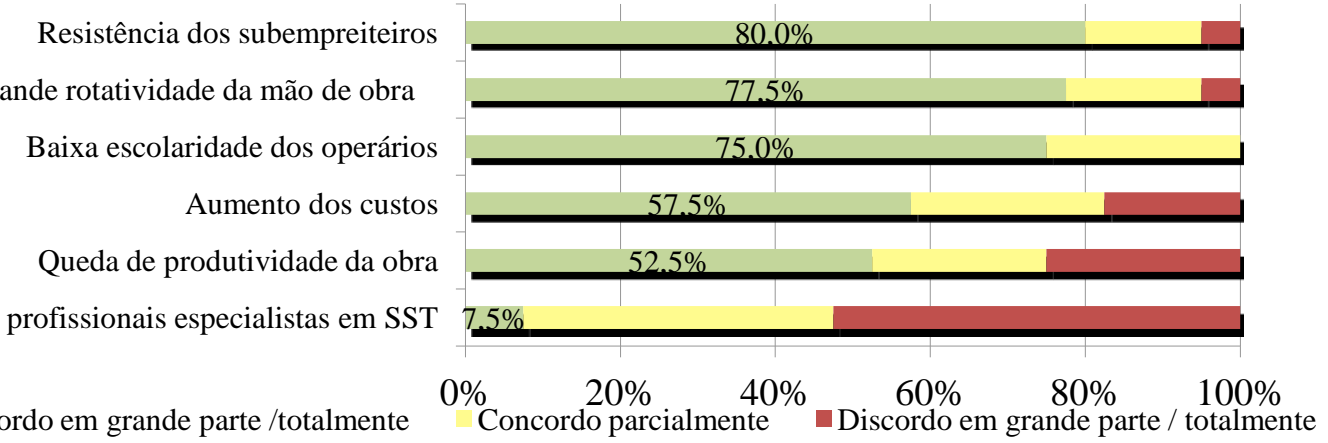

Figura 5 - Dificuldades na implementação das práticas de gestão da SST - Espanha

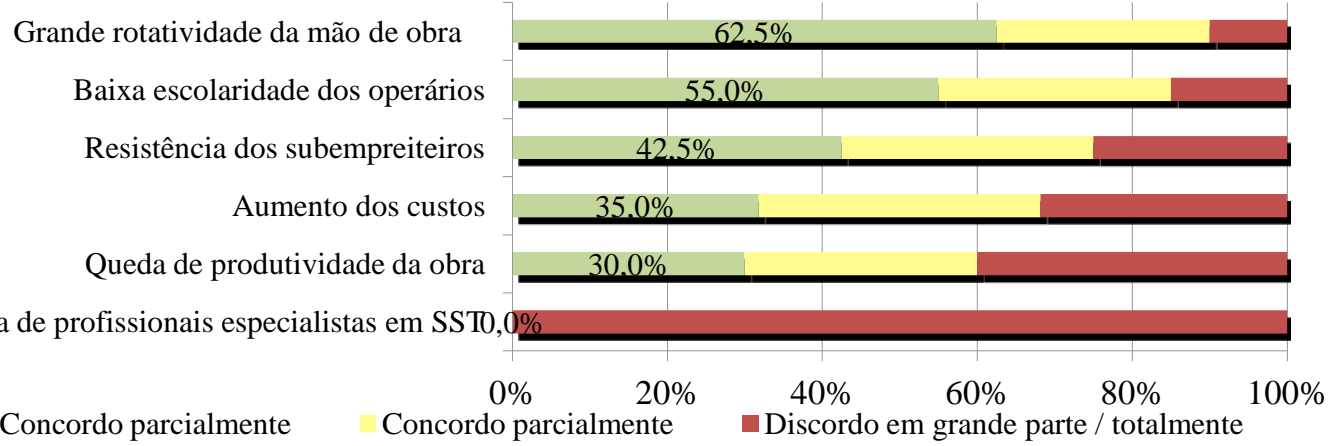

52 Bridi, M. E.; Formoso, C. T.; Pellicer, E.; Fabro, F.; Viguer Castello, M. E.; Echeveste, M. E. S. 
Em ambos os casos os entrevistados discordaram que a falta de profissionais especializados em SST é uma dificuldade para a implantação das práticas de gestão. Esse resultado é coerente com o resultado da importância da "Contratação de Pessoal Especializado", apresentado no item anterior, pois a categoria foi citada como de menor importância em ambos os países.

Ainda, em relação à pergunta aberta, as dificuldades mais citadas pelos entrevistados no Brasil: conscientização e colaboração dos funcionários (citada por 14 entrevistados), prazo (8 citações), mão-de-obra desqualificada e escassa (seis citações), falta de tempo para treinamentos (cinco citações) e pressão por produtividade (quatro citações). E, para os entrevistados da Espanha: a falta de cultura preventiva (14 citações), a priorização da produção (12 citações) e a falta de orientações iniciais aos trabalhadores (10 citações).

Esses resultados indicam que, tanto em relação às dificuldades na implementação das práticas, como no cumprimento da legislação, foi enfocada pelos entrevistados a priorização da produtividade (Brasil e Espanha). Porém, as três dificuldades apontadas como mais importantes no Brasil e na Espanha dizem respeito a problemas relacionados à mão de obra, conforme indica as Quadro $2 \mathrm{e}$ Figura 7. Estes dados indicam uma visão ultrapassada por parte dos responsáveis pela SST em culpar o trabalhador pelas dificuldades relacionadas com a SST. Parece haver uma incoerência na gestão da SST, no sentido de que os trabalhadores são apontados como um empecilho ao cumprimento das normas e na implementação de algumas práticas, enquanto a análise das práticas implementadas demonstra que as práticas que envolvem a mão-de-obra são as que menos são implementadas.

\section{Dificuldades no cumprimento das normas de SST}

Já em relação às dificuldades no cumprimento das normas de SST, apresentadas nas Figura 6 e Quadro 2, a principal dificuldade citada foi o comportamento dos funcionários nas obras (1), com $68 \%$ de concordância no Brasil e $63 \%$ na Espanha, enquanto a ideia de que "alguns itens das normas de segurança inviabilizam a execução de tarefas" (2) obteve o menor índice de concordância com apenas $25 \%$ no Brasil e, no caso da Espanha, foi apontada a queda da produtividade (6), com $23 \%$ de concordância.

\section{Figura 6 - Concordância em relação às dificuldades no cumprimento das normas no Brasil}
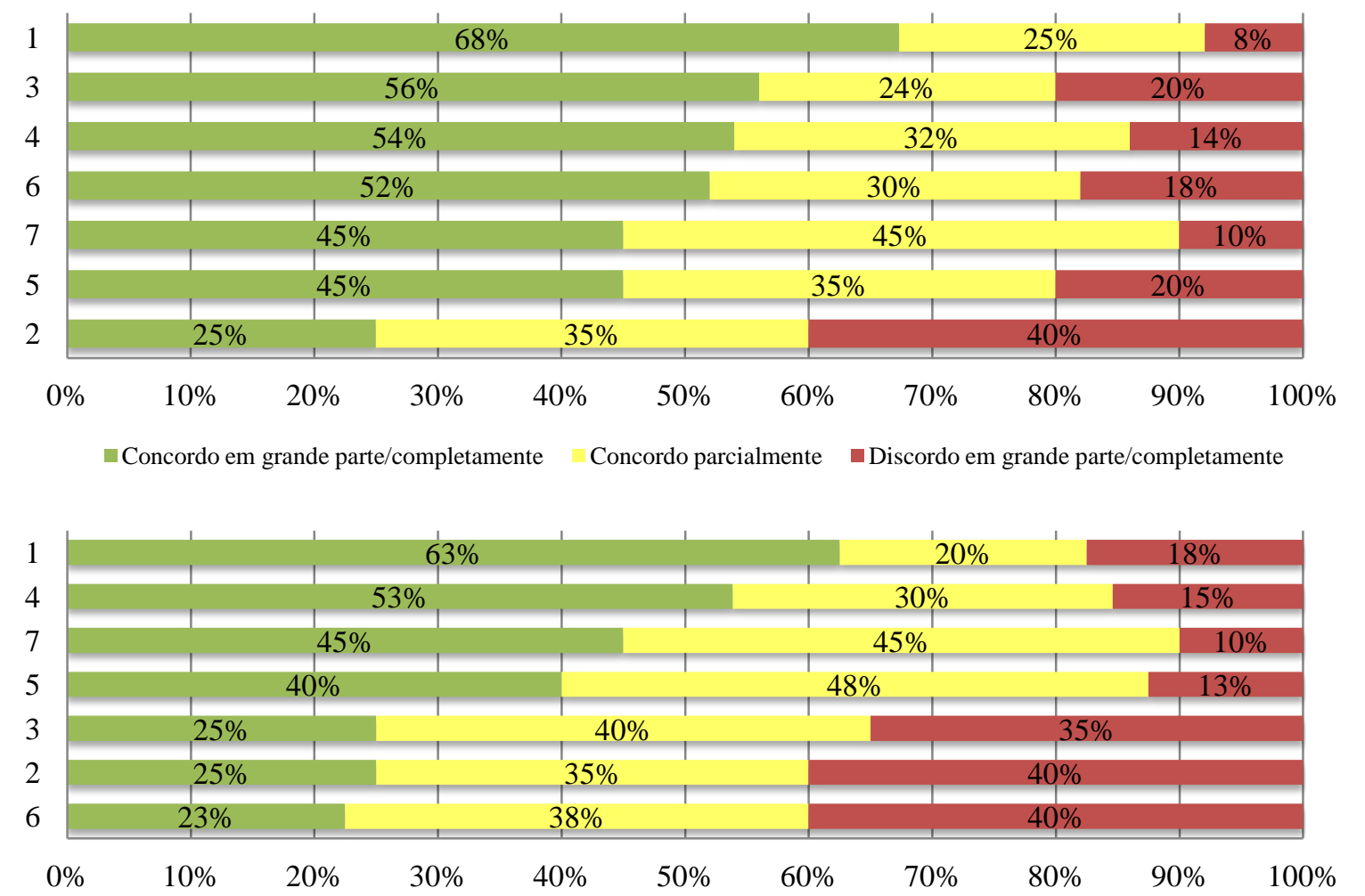


\section{Quadro 2 - Dificuldades no cumprimento das normas na Espanha}

\begin{tabular}{|l|}
\hline 1 O comportamento dos funcionários das obras é uma dificuldade para cumprimento das normas de \\
segurança
\end{tabular}

Assim como foi verificado na pergunta aberta, o comportamento dos funcionários foi apontado como a maior dificuldade no cumprimento da legislação, reforçando a necessidade das empresas em melhorarem as ações gerencias e as práticas com ênfase na participação, no treinamento e na conscientização da mão de obra no canteiro.

\section{Principais causas de multas, interdições e embargos}

No caso do Brasil foram relacionados os itens da NR 18 (ABNT, 2012b), que são apresentados na Figura 7 e na Espanha, a ferramenta foi adaptada para o Título IV da Resolução de $1^{\circ}$ de agosto de 2007 da Regional do Trabalho (DIRECCIÓN..., 2007) (Figura 7), que dispõe sobre as condições mínimas de segurança e saúde aplicáveis às obras de construção.

Pela análise do gráfico de Pareto é possível verificar que no caso brasileiro seis dos 25 itens relacionados são responsáveis por quase metade das causas de multas, interdições e embargos:

(a) medidas de proteção contra quedas em altura;

(b) andaimes e plataformas;

(c) máquinas e equipamentos diversos;

(d) projetos de EPC's;

(e) carpintaria; e

(f) documentação de terceiros.

Em relação à Espanha, quase $80 \%$ das exigências e sanções por parte da Inspeção do Trabalho e da Autoridade do Trabalho são devidos a seis itens: proteção contra o risco de queda em altura, equipamentos de proteção coletiva, controle da documentação própria e de terceiros, andaimes, equipamentos e maquinários e operações envolvendo movimentação de terra.

Assim, os resultados indicam que a maior parte dos itens com maior incidência são similares em ambos os países. Ou seja, o levantamento das principais causas de embargos, interdições e notificações indica que seis itens da norma são responsáveis por $50 \%$ dos problemas das empresas brasileiras e $80 \%$ no caso espanhol, indicando que o controle desse tipo de dado pode permitir que as empresas enfoquem suas ações preventivas nos problemas prioritários.

\section{Conclusões}

Em vista da crescente preocupação da indústria da construção civil brasileira com as questões relacionadas à SST, a investigação realizada pode contribuir tanto para a disseminação das boas práticas de gestão da segurança como para a identificação das dificuldades encontradas pelas empresas, servindo como base para a proposição de melhorias e campanhas enfatizando os aspectos causadores das dificuldades para o setor. Ainda, o principal resultado deste estudo foi o levantamento das práticas de gestão da SST no setor da construção civil, que serviu como base para a realização de um estudo mais aprofundado dessas práticas.

Além disso, embora sejam necessárias adaptações na ferramenta de coleta ao se aplicar em outros contextos, no que diz respeito à correta tradução e compatibilização e às legislações específicas, como foi o caso da Espanha, sugere-se a realização deste tipo de pesquisa em outros países iberoamericanos, que apresentam algumas similaridades ao Brasil no que se refere a práticas de gestão da SST.

54 Bridi, M. E.; Formoso, C. T.; Pellicer, E.; Fabro, F.; Viguer Castello, M. E.; Echeveste, M. E. S. 
Figura 7 - Itens causadores de multas, interdições e embargos nas obras no Brasil

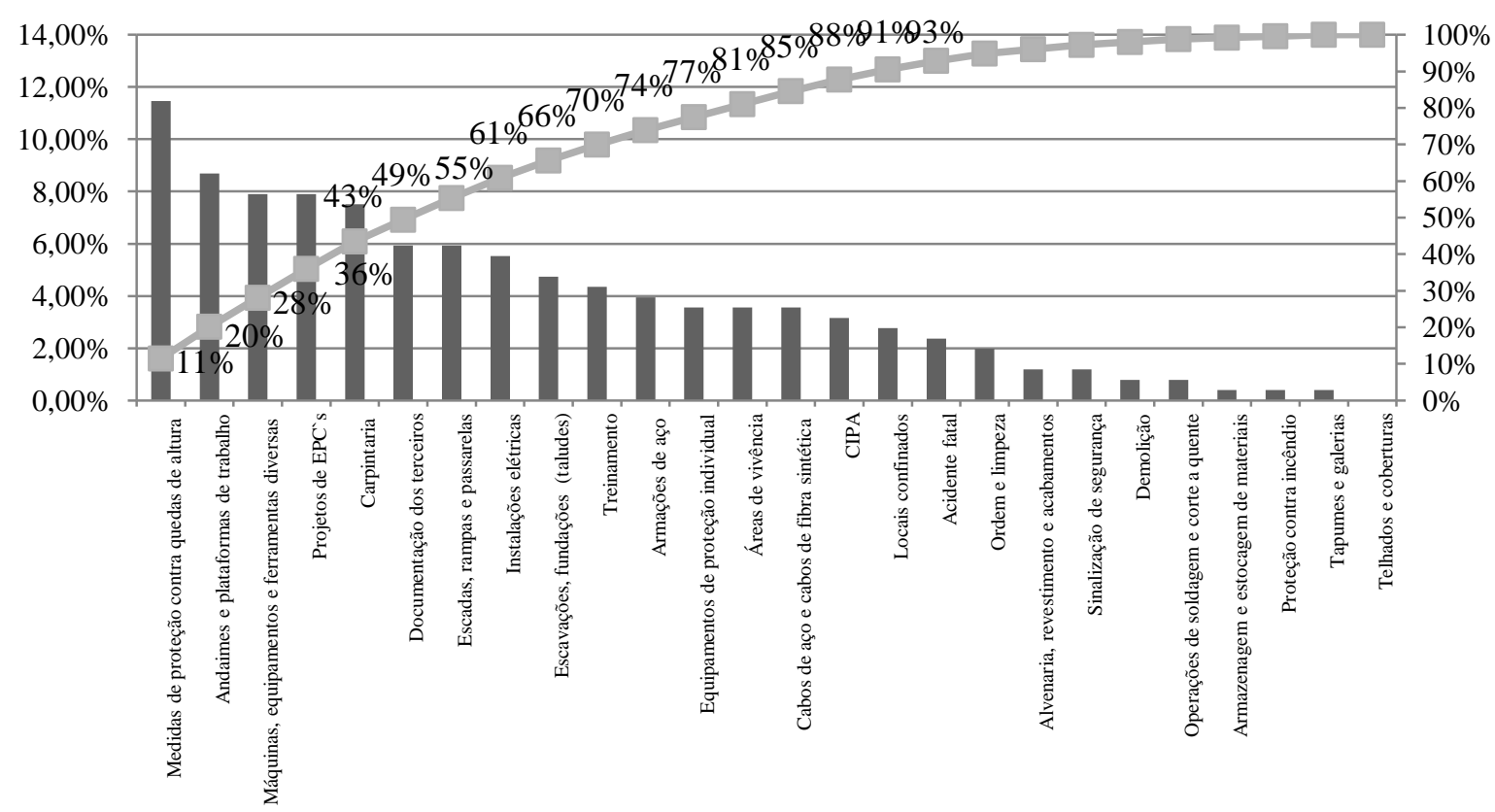

Figura 8 - Itens causadores de multas, interdições e embargos nas obras na Espanha

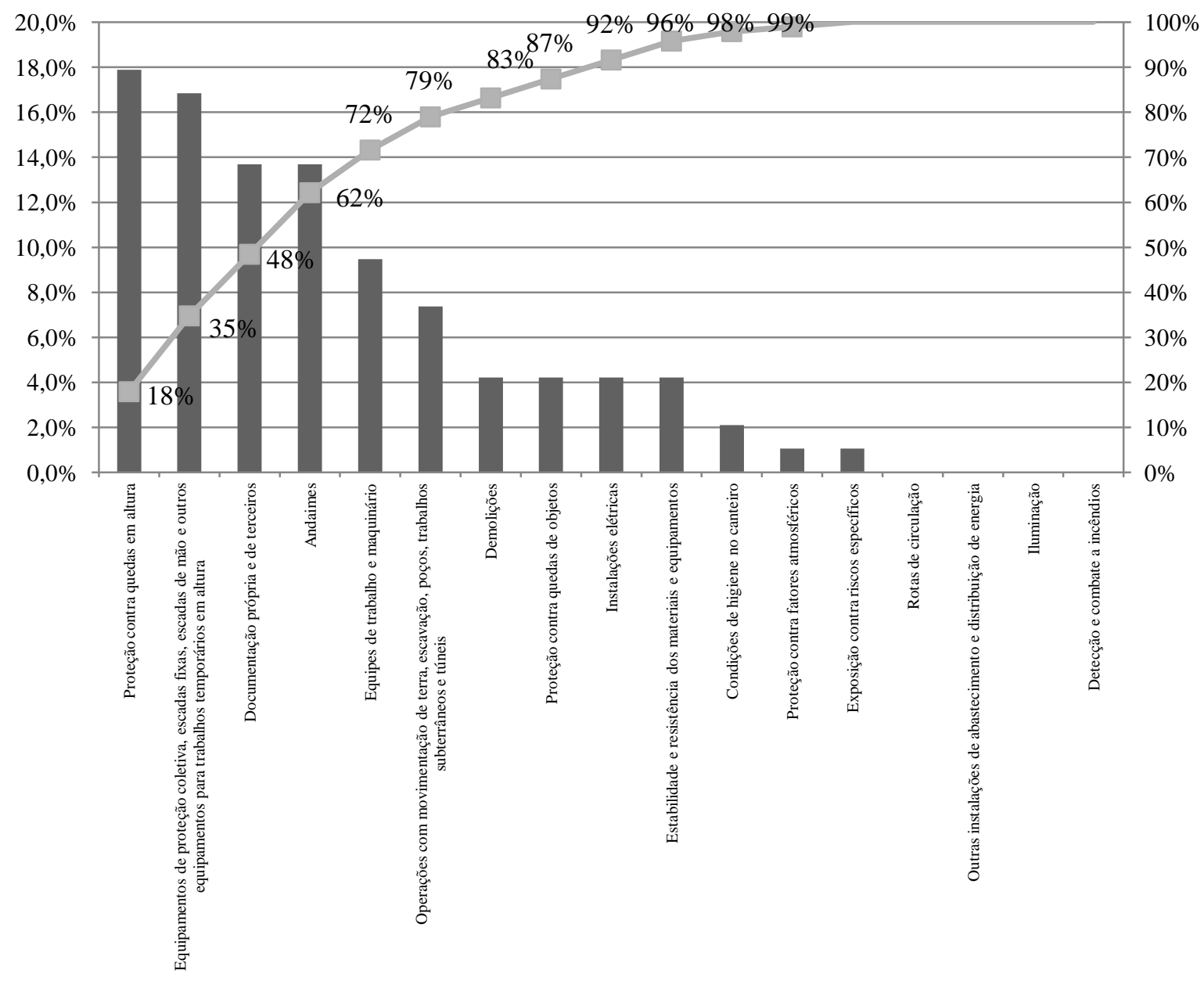


Esta pesquisa poderia ser também ampliada para outros segmentos da indústria da construção civil, a fim de verificar, de forma comparativa, a relação do contexto organizacional com a utilização das práticas. Também, sugere-se a realização de uma comparação entre os índices de utilização das práticas com os indicadores tradicionais de acidentes, de caráter reativo, tais como a taxa de frequência e gravidade de acidentes, a fim de analisar o impacto da utilização dessas práticas na melhoria do desempenho em SST.

\section{Referências}

ABUDAYYECH, O.; FREDERICKS, T.; BUT, S.; SHAAR, A. An Investigation of Management's Commitment to Construction Safety, International Journal of Project Management, v. 22, p.167-174, 2006.

ASFAW, B.; PANA-CRYAN R.; ROSA, R. The Business cycle and the incidence of workplace injuries: evidence from the U.S.A. Journal of Safety Research, v. 42, p. 1-8, 2011.

BRASIL. Embargo e Interdição: a experiência da seção de segurança e saúde no trabalho:

SEGUR/RS. Porto Alegre: Ministério do Trabalho e Emprego, 2010.

BRASIL. Ministério do Trabalho. Dados da Inspeção em Segurança e Saúde no Trabalho 2012. Disponível em: <http://portal.mte.gov.br/data/files/8A7C812D3D1 83EB0013D2621437B3960/Acumulado\%20$\% 20$ DSST\%20-\%20\%202012.pdf>. Acesso em: 20 set. 2013.

BRASIL. Ministério do Trabalho. NR 18: condições e meio ambiente de trabalho na indústria da construção. 2012.

BRIDI, M.E. Protocolo de Avaliação das Práticas de Segurança e Saúde no Trabalho no Setor da Construção Civil. Porto Alegre, 2012. Dissertação (Mestrado em Engenharia Civil) Programa de Pós-Graduação em Engenharia Civil, Universidade Federal do Rio Grande do Sul, Porto Alegre, 2012.

BRITISH STANDARDS INSTITUTION. OHSAS 18001: occupational health and safety management systems. Londres: BSI, 2007. Occupational Health and Safety Assessment Series.

CAMBRAIA, F. B.; FORMOSO, C. T. Análise de Avanços e Retrocessos no Atendimento às Especificações da NR-18 nos Últimos Dez Znos. In: SIMPÓSIO BRASILEIRO DE GESTÃO E ECONOMIA DA CONSTRUÇÃO, 7., Belém do Pará, 2011. Anais... Belém do Pará, 2011.
DIAS, L.M.; COBLE, R., Construction Safety Coordination in the European Union. Lisbon, 1999. CIB Publication 238 W99.

DÍAZ, C.; ORDEN, M.V.; ZIMMERMANN, M. Actividades Económicas Con Mayor Siniestralidad, Penosidad y Peligrosidad: sector de la construcción. Madrid: Departamento de Investigación e Información, 2010.

DIRECCIÓN GENERAL DE TRABAJO. IV Convenio Colectivo General del Sector de la Construcción. Boletín Oficial del Estado, Madrid, v. 197, 2007.

EVERETT, J.; THOMPSON, W. Experience Modification Rating For Workers' Compensation Insurance. Journal of Construction Engineering and Management, v. 121, n.1, p. 66-79, 1995.

EUROPEAN COMMISSION. Non-Binding Guide to Good Practice For Understanding and Implementing Directive 92/57/EEC: on the implementation of minimum safety and health requirements at temporary or mobile construction sites. Luxembourg: Publications Office of the European Union, 2011.

FAMÁ, C. C. G. Diretrizes Para Avaliação de Sistemas de Medição de Desempenho na SST no Setor da Construção Civil. Porto Alegre, 2010. Dissertação (Mestrado em Engenharia Civil) Programa de Pós-Graduação em Engenharia Civil, Universidade Federal do Rio Grande do Sul, Porto Alegre, 2010.

FANG, D. et al. Factor Analysis-Based Studies on Construction Workplace Safety Management in China. International Journal of Project Management, v. 22, n. 1, p. 43-49, 2004.

FORMOSO, C. T.; PELLICER, E.; YEPES, V. Occupational Safety and Health in Construction: some international experiences on education and training. In: INTERNATIONAL TECHNOLOGY, EDUCATION AND DEVELOPMENT CONFERENCE, 5., Valencia, 2011. Proceedings... Valencia, 2011. p. 6530-6536.

FUNG, I. et al. Safety Cultural Divergences Among Management, Supervisory and Worker Groups in Hong Kong Construction Industry. International Journal of Project Management, v. 23, n. 7, p. 504-512, 2005.

GYI, D.; GIBB, A.; HASLAM, R., The Quality of Accident and Health Data in the Construction Industry: interviews with senior managers. Construction Management and Economics, v. 17, p. 197-204, 1999.

56 Bridi, M. E.; Formoso, C. T.; Pellicer, E.; Fabro, F.; Viguer Castello, M. E.; Echeveste, M. E. S. 
HARPER, R.; KOEHN, E. Managing Industrial Construction Safety in Southeast Texas. Journal of Construction Engineering and Management, v. 124 , n. 6 , p. 452-457, nov./dec. 1998.

HINZE, J. Making Zero Injuries a Reality: a report to the construction industry institute. Gainesville: University of Florida, 2002. Report 160 .

HUANG, X.; HINZE, J. The Owner's Role in Construction Safety. Journal of Construction Engineering and Management, v. 132, n. 2, p.164-173, 2006a.

HUANG, X.; HINZE, J. The Owner's Role in Construction Safety: Guidance Model, Journal of Construction Engineering and Management, v. 132, n. 2, p. 174-181, 2006b.

JASELSKI, E.; ANDERSON, S.; RUSSEL, J. Strategies For Achieving Excellence in Construction Safety Performance. Journal of Construction Engineering and Management, v. 122, n. 1, p. 61-70, 1996.

KATO, R. M.; SERRA, S.M.B. Execução de PréMoldados de Concreto Considerando Aspectos da Segurança e Saúde do Trabalho (SST) Segundo a Engenharia de Resiliência (ER). In: Encontro Nacional de Tecnologia do Ambiente Construído, 14., Juiz de Fora, 2012. Anais... Juiz de Fora, 2012. v. 1.

LAI, D. N. C.; LIU, M.; LING, F. Y. Y. A Comparative Study on Adopting Human Resource Practices For Safety Management on Construction Projects in the United States and Singapore. International Journal of Project Management, v. 29, n. 8, p. 1018-1032, dec. 2011.

LISKA, R.W.; GOODLOE, D.; SEN, R. Zero Accident Techniques. Austin: The Construction Industry Institute, 1993.

MALHOTRA, N. Pesquisa de Marketing: uma orientação aplicada. 4. ed. Porto Alegre: Bookman, 2006.

MALlmann, B. S. Avaliação do Atendimento aos Requisitos da NR 18 em Canteiros de Obra. Porto Alegre, 2008. Graduação (Trabalho de Conclusão de Curso em Engenharia Civil) Departamento de Engenharia Civil, Universidade Federal do Rio Grande do Sul, Porto Alegre, 2008.

MARTINEZ, M. D.; RUBIO, M. C.; GIBB, A. Prevention Through Design: the effect of european directives on construction workplace accidents. Safety Science, v. 48, n. 2, p. 248-258, 2010.
MELO, E. H.; SANTOS, C. R. F. G..; SERRA, S. M. B. Sistemas de Proteção Coletiva: boas práticas e normas técnicas. In: ENCONTRO NACIONAL DE TECNOLOGIA DO AMBIENTE CONSTRUÍDO, 14., Juiz de Fora, 2012. Anais... Juiz de Fora, 2012.

MOHAMED, S. Safety Climate in Construction Site Environments. Journal of Construction Engineering and Management, v. 128, n. 5, p. 375-384, 2002.

POTTS, S.; MCGLOTHLIN, J. D. Analysis of Safety Programs of 16 Large Construction Companies. School of Health Sciences Purdue University, 2003.

RAZURI, C. Un Sistema Integrado de Gestión de Producción y Seguridad en La Construcción. Santiago, 2007. Tesis (Magíster en Ciencias de la Ingeniería) - Escuela de Ingeniería, Universidad Católica de Chile, Santiago, 2007.

REESE, E. Handbook of OSHA Construction Safety and Health, New York: Lewis Publishers, 1999.

ROCHA, C. A. G. Diagnóstico do Cumprimento da NR-18 no Subsetor Edificações da Construção Civil e Sugestões para Melhorias. Porto Alegre, 1999. Dissertação (Mestrado em Engenharia Civil) - Programa de Pós-Graduação em Engenharia Civil, Universidade Federal do Rio Grande do Sul, Porto Alegre, 1999.

ROWLINSON, S. Human Factors in Construction Safety Management Issues. In: COBLE, R.; HINZE, J., HAUPT, T. (Eds.). Construction Safety and Health Management. Prentice-Hall: Upper Saddle River, 2000. p. 59-83.

SAURIN, T. A. Método Para Diagnóstico e Diretrizes Para Planejamento de Canteiros de Obra de Edificações. Porto Alegre, 1997. Dissertação (Mestrado em Engenharia Civil) Escola de Engenharia, Universidade Federal do Rio Grande do Sul, 1997.

SAURIN, T.A. Segurança e Produção: um modelo para o planejamento e controle integrado. Porto Alegre, 2002. Tese (Doutorado em Engenharia de Produção) - Programa de PósGraduação em Engenharia de Produção, Universidade Federal do Rio Grande do Sul, Porto Alegre, 2002.

SAWACHA, E.; NAOUM, S.; FONG, D. Factors Affecting Safety Performance on Construction Sites. International Journal of Project Management, v. 17, n. 5, p. 309-315, 1999.

SERRA, S. M. B. Proteção nas Alturas. Techne: Revista de Tecnologia da Construção, São Paulo, p. 28-31, jul. 2010. 
TEO, E. A. L.; LING, F. Y. Y.; CHONG, A. F. W. Framework For Project Managers to Manage Construction Safety. International Journal of Project Management, v. 23, n. 4, p. 329-341, 2005.

WINPEPI. Computer Programs For Epidemiologists. SPSS 11. IBM: Chicago, Illinois; 2006.

\section{Agradecimentos}

Os autores agradecem ao CYTED e ao CNPq pelo financiamento da pesquisa, e também aos colegas da Universidade de la República (Uruguai), Pontifícia Universidad Católica de Chile, Universidad de los Andes (Colômbia), Instituto Tecnológico e de Estudos Superiores de Monterrey (México), Universidad de Granada (Espanha), Universidade Técnica de Lisboa e Universidade do Minho (Portugal), e Universidade Federal de São Carlos (UFSCar), pelas contribuições feitas à pesquisa.

\footnotetext{
Marcelle Engler Bridi

Departamento de Engenharia Civil, Escola de Engenharia | Universidade Federal do Rio Grande do Sul | Av. Osvaldo Aranha, 99, $3^{\circ}$ andar, Farroupilha | Porto Alegre - RS - Brasil | CEP 90035-190 | Tel.: (051) 3316-3518 | E-mail: marcelle.bridi@gmail.com
}

\section{Carlos Torres Formoso}

Departamento de Engenharia Civil, Escola de Engenharia | Universidade Federal do Rio Grande do Sul | E-mail: formoso@ufrgs.br

\section{Eugenio Pellicer}

Universitat Politècnica de València | Camino de Vera, s/n | Valencia - Espanha | 46022 | Tel. +(34) 9638-79562

E-mail: pellicer@upv.es

\section{Fabiana Fabro}

Departamento de Engenharia Civil, Escola de Engenharia | Universidade Federal do Rio Grande do Sul |

E-mail: fabiana.engcivil@gmail.com

\section{Maria Encarnacion Viguer Catello}

Universitat Politècnica de València | E-mail: mavicas@cst.upv.es

\section{Marcia Elisa Soares Echeveste}

Departamento de Estatística, Instituto de Matemática | Universidade Federal do Rio Grande do Sul | Av. Oswaldo Aranha, 99, 508, Centro Porto Alegre - RS - Brasil | CEP 90035-190 | Tel.: (51) 3308-4297 | E-mail: echeveste@producao.ufrgs.br

\section{Revista Ambiente Construído}

Associação Nacional de Tecnologia do Ambiente Construído

Av. Osvaldo Aranha, $99-3^{\circ}$ andar, Centro

Porto Alegre - RS - Brasil

CEP $90035-190$

Telefone: +55 (51) 3308-4084

Fax: +55 (51) 3308-4054

www.seer.ufrgs.br/ambienteconstruido

E-mail: ambienteconstruido@ufrgs.br 